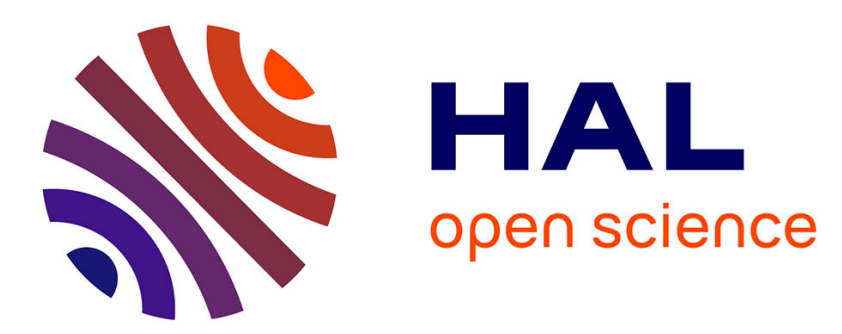

\title{
Locating Multiple Soft Faults in Wire Networks Using An Alternative DORT Implementation
}

\author{
Moussa Kafal, Andrea Cozza, Lionel Pichon
}

\section{To cite this version:}

Moussa Kafal, Andrea Cozza, Lionel Pichon. Locating Multiple Soft Faults in Wire Networks Using An Alternative DORT Implementation. IEEE Transactions on Instrumentation and Measurement, 2016, 65 (2), pp.399-406. 10.1109/TIM.2015.2498559 . hal-01198796

\section{HAL Id: hal-01198796 https://hal.science/hal-01198796}

Submitted on 14 Sep 2015

HAL is a multi-disciplinary open access archive for the deposit and dissemination of scientific research documents, whether they are published or not. The documents may come from teaching and research institutions in France or abroad, or from public or private research centers.
L'archive ouverte pluridisciplinaire HAL, est destinée au dépôt et à la diffusion de documents scientifiques de niveau recherche, publiés ou non, émanant des établissements d'enseignement et de recherche français ou étrangers, des laboratoires publics ou privés. 


\title{
Locating Multiple Soft Faults in Wire Networks Using An Alternative DORT Implementation
}

\author{
Moussa Kafal, Student Member, IEEE Andrea Cozza, Senior Member, IEEE and Lionel Pichon, Member, IEEE
}

\begin{abstract}
Decomposition of the time reversal operator (DORT) was recently applied to the problem of detection and location of soft faults in wire networks and proved effectual when dealing with a single fault, even in the case of complex network configurations. In this paper, the case of location of multiple faults is addressed, first proving that the standard DORT formulation does not allow to take a clear decision about the individual position of each fault. An alternative version of the DORT, based on an updating procedure, is presented and demonstrated to enable accurate and selective location of multiple soft faults. The proposed procedure is also shown to allow estimating the reflection coefficient of each fault, thus giving access to their severity.
\end{abstract}

Index Terms-Fault location, soft faults, multiple faults, complex wire networks, DORT imaging.

\section{INTRODUCTION}

Electrical cables are infrastructures widely used for distributing power and communication signals throughout nearly all modern systems [1]. Their cumulative length can exceed hundreds of kilometers in many networks employed in work and transportation, e.g., $5000 \mathrm{~km}$ in power plants, $500 \mathrm{~km}$ in large aircrafts and $4 \mathrm{~km}$ in modern cars [2].

As any system, cable networks are subject to wearing and accidents that can make them nonoperational. Open and short circuits occurring along a cable are the most well-known sources of faults; they are usually referred to as hard faults, as they stop signals from propagating further along a cable. As opposed to them, soft faults [3] feature minor alterations, such as insulation wearing; in this case, signals can still propagate along a cable, and a network is in no danger of breaking down. Yet, a partial wearing may evolve into a stronger degradation, eventually leading to a hard fault [4]. Hence, developing robust procedures for the identification and location of soft faults becomes important, as an early-warning approach for ensuring the safe operation of a critical infrastructure. As a matter of fact, studies conducted on cables showed that $30 \%$ to $50 \%$ of detected wiring faults are considered to be soft.

Despite the fact that several techniques have been introduced throughout the last decades to detect and locate faults, reflectometry-based methods are still the most widely used, without having much changed in their scope and definition [5]. The state-of-art of reflectometry for wiring fault detection can be categorized into two main families: Time Domain Reflectometry (TDR) [6], [7], [8] and Frequency Domain Reflectometry [9], [10]. Basically, both methodologies are based on an appropriate analysis of the reference and the reflected

M. Kafal, A. Cozza and L. Pichon are with GeePs, UMR 8507, CENTRALESUPELEC - Univ Paris-Sud - CNRS, UPMC, 3 rue Joliot-Curie, 91192 Gif-sur-Yvette, France. Contact e-mail: andrea. cozza@ieee. org signal either in the time or frequency domain. Besides, they are known to perform well when dealing with hard faults, which result in large reflections, but start failing with soft ones. In fact, soft faults are characterized by a very weak reflectivity, which produce echoes that can pass unnoticed compared to those caused, e.g., by junctions within a network under test (NUT) [3], [4], [11], [12]. A recent work [13] aiming at locating multiple faults falls in this same group, as it mainly works for relatively hard faults.

Based on a radically different concept, the DORT method, standing for decomposition of the time reversal operator, was originally developed in acoustics as a remote-sensing technique for the detection and location of scatterers within homogeneous media [14]. Basically, it allows defining signals bound to focus over one of the scatterers, thus providing a visual method for inferring the position of a previously unknown scatterer. This idea was first transposed to guidedwave propagation, e.g., wire networks, in [15] and shown to precisely detect and locate single soft faults even within complex network configurations, involving a number of junctions. The proposed method presented major advantages compared to TDR-based techniques where the eventual presence of a fault is inferred from the measured scattering matrix of the NUT, rather than from echoes, as done in TDR methods. Besides, it has allowed the simultaneous analysis of all multiport measured data which are jointly processed to locate the actual position of the fault [15], as opposed to methods exploiting multiport data separately [16].

While passing from a single scatterer to several is trivial with waves propagating in homogeneous media, we show in this paper that in the case of wire networks this is no longer true. Multiple faults cannot be resolved separately, because of their strong coupling via guided propagation. An alternative formulation of the DORT based on an updating scheme is proposed and proved to allow selective focusing and location of multiple soft faults.

The paper starts by recalling the methodology of the standard DORT technique, hereafter referred to as SDORT, in locating single soft faults, in particular the main steps followed in measuring the NUT's response and the subsequent faultdetection procedure. After that, section III will be devoted to explaining the limitations SDORT faces when multiple faults occur. This is followed by presenting a solution characterized by separating the measured NUT's data allowing locating each fault selectively, thanks to a mathematical model discussed in section IV-A. The new procedure is detailed in section IV-B and will be referred to as the enhanced DORT (EDORT) method. As a by product, it allows retrieving the faults' intensities, as demonstrated in section IV-C, an important 
feature when needing to decide if a fault should be checked more closely by a human operator or just in order to monitor how the fault evolves in time. Experimental results dealing with coaxial cables are provided to validate the proposed method.

\section{STANDARD DORT FOR FAULT LOCATION}

The basic operations and properties involved in the use of the SDORT technique in fault location are here recalled; the interested reader should refer to [15] for more details. All subsequent quantities are functions of the frequency, except where otherwise indicated, hence the frequency variable will be dropped.

When dealing with an NUT displaying $N$ testing ports, its scattering matrix $\mathbf{S}$ allows a direct computation of the output signals generated by any testing signal applied to one or more of its $N$ testing ports. As such, SDORT applied to fault detection is based on the availability of this matrix, which can be measured, as discussed in sec. V-A, by means of a vector network analyzer (VNA). As it is often the case when dealing with soft faults, the procedure described in [15] requires a baselining approach, i.e., taking the difference between the response of the NUT, containing an eventual fault, and a reference response of a healthy version of it [3] [17]. This operation ideally removes the spurious echoes generated by impedance discontinuities like junctions, leaving only those echoes initially generated by the interaction between the testing signals and the faults. For the case of SDORT, the NUT response after baselining will be $\boldsymbol{S}=\boldsymbol{S}_{f}-\boldsymbol{S}_{h}$, with $\boldsymbol{S}_{f}$ and $\boldsymbol{S}_{h}$ being the scattering matrices of the (eventually) faulty NUT and its healthy version, respectively.

Following the properties of the SDORT in free-space propagation, sets of input signals can be defined, such that when injected through the testing ports they will lead to waves focusing onto each fault. These signals can be computed by solving the eigenproblem $\boldsymbol{S} \boldsymbol{S}^{H} \boldsymbol{U}_{n}=\lambda_{n} \boldsymbol{U}_{n}$, where $H$ is the Hermitian transpose. The number $n<N$ of most significant eigenvalues hints at the number of potential faults found in the NUT; if the properties proved for SDORT [14] hold also for NUTs, then each fault could be spotted by monitoring the propagation of input signals whose Fourier spectra would be defined by the scalar components of each eigenvector $\boldsymbol{U}_{n}$. This operation can be carried out by means of a numerical simulator for transmission lines, modelling the layout of the healthy NUT; the fault's position would be found by looking for maximal energy focusing. To this end, the total energy $E(x)=\int v^{2}(x, t) d t$ can be computed from the voltage $v(x, t)$ observed throughout the numerical model of the NUT.

The theoretical separability of each fault response would also imply the possibility of inferring their respective severity, with clear practical implications in early-wake monitoring of a wire network. The discussions in the next section prove that this feature is not possible with SDORT; this notwithstanding, the procedure proposed in section IV will be shown to reinstate this property.

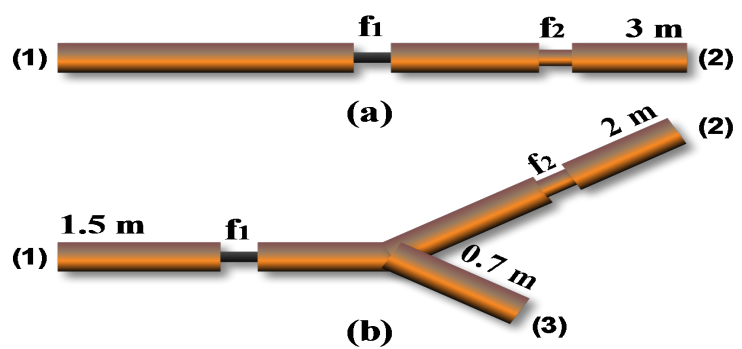

Fig. 1: Layouts of the NUTs considered in the numerical and experimental studies: (a) single-branch NUT; (b) singlejunction network configuration. Both structures include the lengths of the branches and present two soft faults of different severity.

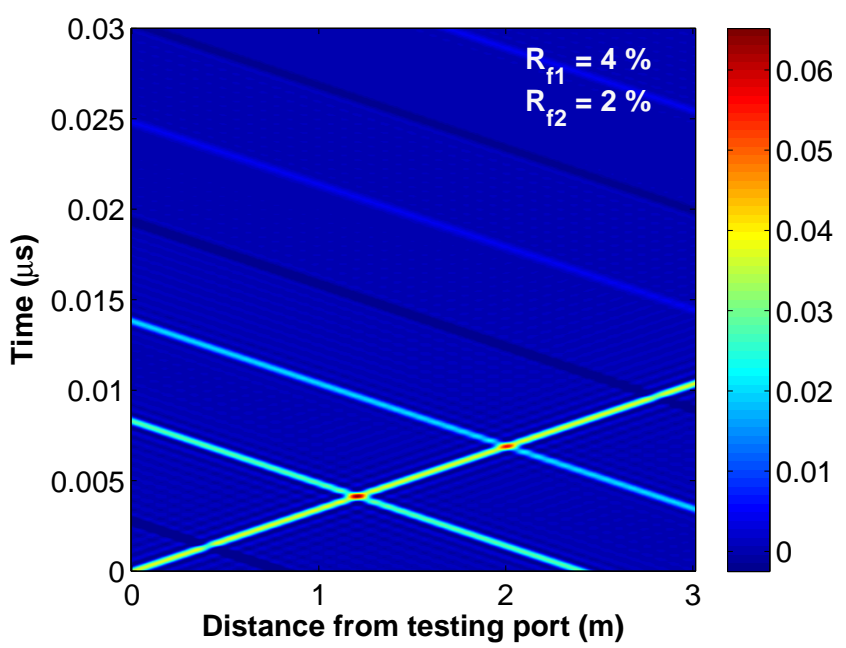

Fig. 2: The space-time (ZT) diagram showing voltage propagation along the single-branch NUT in Fig. 1(a). Two focal spots of different intensity can be observed at the positions of the two faults, at 1.15 and 2 meters.

\section{SDORT PERFORMANCE WITH MULTIPLE FAULTS}

The limitations SDORT faced in case of multiple faults can be easily exposed by means of numerical simulations. Those shown in this paper are based on the use of an in-house code that solves networks of cables, connected by means of junctions and lumped loads; standard transmission-line theory is used to this end [18].

Numerical simulations were carried out for the NUTs presented in Fig. 1, where two different layouts were considered: a single-branch and a single-junction structure. Both involve uniform two-wire transmission lines, with the two conductors $10 \mathrm{~mm}$ apart, each with a $1 \mathrm{~mm}$ radius and with a $0.5 \mathrm{~mm}$ dielectric coating of relative permittivity $\epsilon_{r}=3$.

In order to identify unambiguously a portion of the NUT of interest in our discussions, a number is assigned to each of the terminations serving as testing ports. We set number (1) as the origin, and distances will be measured with respect to it, and consequently all graphs will be plotted accordingly, with this distance as the independent variable. We will be interested in 


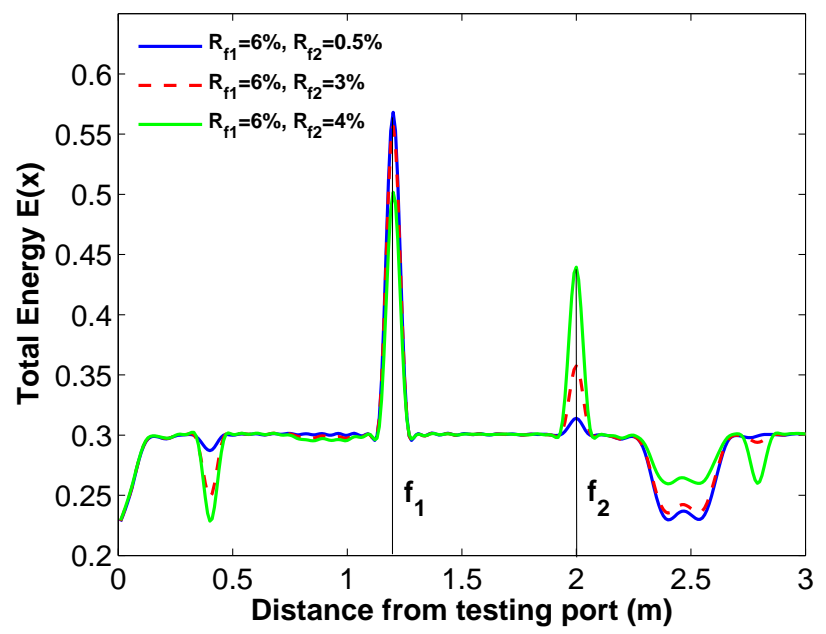

Fig. 3: The total energy $E(x)$ observed along the NUT in Fig. 1(a) with two soft faults at $1.15 \mathrm{~m}$ and $2 \mathrm{~m}$ from the origin port comparing three different sets of fault severities.

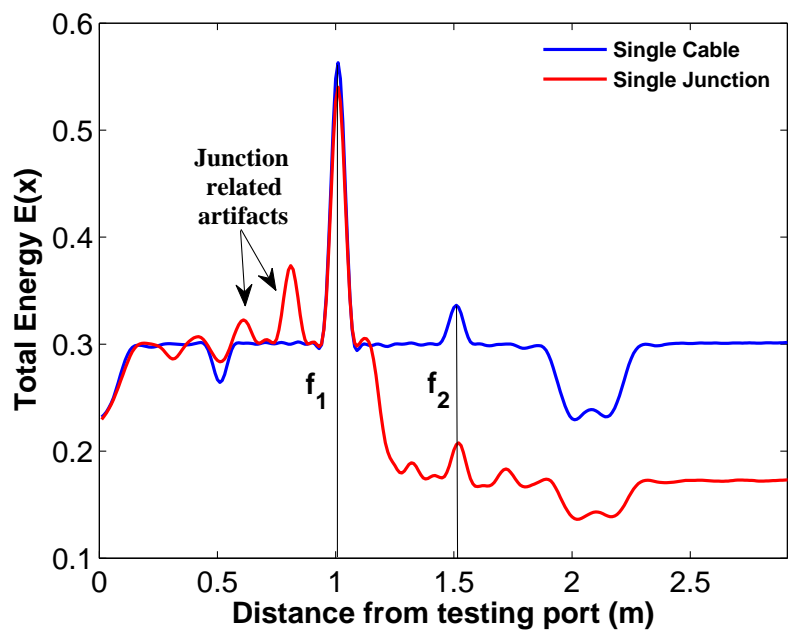

Fig. 4: The total energy $E(x)$ obtained for the description comparing the responses of weak faults with respect to the network's complexity between the single-branch configuration of Fig. 1(a) and the single-junction NUT of Fig. 1(b) along its second path, while considering two soft faults at the same distances with respect to the reference port $(1 \mathrm{~m}$ for the first soft fault and $1.5 \mathrm{~m}$ for the weaker one).

observing signals propagating along portions of the NUT; for the sake of simplicity these propagation paths will originate from port (1), the origin, and end at another testing port. A path can therefore be identified by referring to the far-end testing port. As an example, in Fig. 1(b), the third path is the part of the network linking the origin to the testing port numbered (3).

The lengths of the wires composing the paths are chosen in accordance to the lengths of cables available for the experimental setup discussed in sec. V. Soft faults are introduced in the numerical models by removing a portion of the coating of the lines, which can model a potential pressure on the wires or a partial erosion of the coating, while in our experiments we relied on coaxial cables, where coating removal is not an option; in this case we rather crushed a portion of the cable in order to introduce a local impedance discontinuity (see sec. V).

Two such faults are introduced, where the strongest is obtained by reducing the thickness of the coating from $0.5 \mathrm{~mm}$ to $0.05 \mathrm{~mm}$ over a length of $2 \mathrm{~cm}$ thus corresponding to a 0.45 $\mathrm{mm}$ coating removal, whereas the weaker one is associated to a mere $0.05 \mathrm{~mm}$ coating removal. The effect of these local modifications in the coating can be quantitatively assessed by computing the relative change $R_{f}=\left|Z_{f}-Z_{c}\right| /\left|Z_{c}\right|$ measuring the deviation from the characteristic impedance $Z_{c}$ of the line, after introducing a fault of impedance $Z_{f}$.

The first issue with SDORT is the lack of selective focusing. This can be demonstrated by tracking the voltage propagation both in time and space in the NUT, hereafter referred to as ZT diagram, generated by applying to the testing ports the eigenvector corresponding to the most significant eigenvalue $\lambda_{n}$. According to [14], there should be a single-spot appearing at the position of the most significant fault. In fact, for the NUT in Fig. 1(a), the ZT diagram of Fig. 2 shows two focal spots where the faults were introduced.

Even though the faults are not excited individually, it could be argued that they are still visible and rightly located. That is where the second issue comes in: the ratio of the focal-spot intensities does not appear to represent the ratio of the fault severities. This means that it is not possible to use the focalspot intensity as a proxy of a fault severity. Moreover, having focal-spot intensities spanning a large dynamical range can hinder the detection of faults associated to weaker focusing, particularly in the case of noisy signals. As an example, consider the NUT in Fig. 1(a) with two faults of different intensities, placed at $1.15 \mathrm{~m}$ and $2 \mathrm{~m}$, respectively. When choosing a weaker second fault $\left(R_{f 2}\right.$ goes from $4 \%$ down to $0.5 \%)$, while keeping the first one fixed $\left(R_{f 1}=6 \%\right)$, the energy diagrams in Fig. 3 show a vanishingly low focusing over the weaker fault, resulting into a less likely identification. Moreover, the reduction in the energy at the second-fault position does not seem to be related to the reduction in the fault intensity.

One of the most interesting properties of SDORT was its effectiveness in locating single faults within complex NUTs, composed of several junctions and branches [15]. This property is no longer guaranteed in the case of multiple faults. In order to better illustrate this point, the NUTs shown in Fig. 1 were considered, each with two faults where $R_{f 1}=6 \%$ and $R_{f 2}=3 \%$, found at the same distances with respect to the reference port ( $1 \mathrm{~m}$ for the stronger soft fault and $1.5 \mathrm{~m}$ for the weaker one). The objective is to assess how a changing NUT complexity impacts the focusing capabilities of SDORT. The energy diagrams in Fig. 4 show that a more complex NUT introduces an ambiguity on the weaker fault's position, due to artifacts that appear as a result of junction-related echoes, in spite of the baselining procedure. This situation is expected to worsen with NUT's composed of several junctions and branches. 


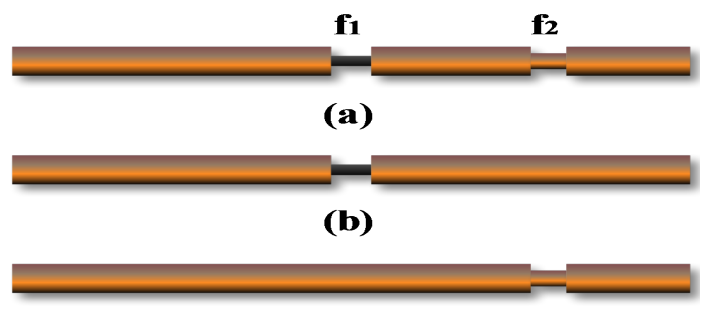

(c)

Fig. 5: A single-branch NUT used for demonstrating the possibility of separating a multiple-fault scattering matrix containing two soft faults (a) into two replicas of the NUT presenting only fault 1 (b) or fault 2 (c).

\section{Alternative iterative FOrmulation}

The issues with SDORT in case of multiple faults can be ascribed to the coupling existing between the faults' responses, which in turn leads to simultaneous multiple focusing. If the signals relative to each fault could be separated, then individual focusing would be available again, making the detection and location of the faults more robust and precise. Moreover, without separation of the fault responses, it is not possible to assess their severity, a major drawback in an early-warning technique.

\section{A. Separation of a scattering matrix}

The key element in addressing separately each fault is to understand how the response an NUT featuring only one fault at the time is related to the response of the multiple-fault case. In order to get a better insight into this problem, we start by considering the simple NUT in Fig. 5(a) consisting of a single-branch with two soft faults with reflection coefficients $\Gamma_{1}$ and $\Gamma_{2}$, respectively. Neglecting multiple reflections, its transmission coefficient can be expressed as

$$
S_{21}^{f}=\left(1+\Gamma_{1}\right)\left(1+\Gamma_{2}\right) e^{-\mathrm{j} \beta L},
$$

where $\beta$ is the propagation constant and $L$ the total length of the line, whereas for the case of the healthy configuration

$$
S_{21}^{h}=e^{-\mathrm{j} \beta L} .
$$

Hence the baselined difference scattering transmission coefficient is

$$
\begin{aligned}
S_{21} & =S_{21}^{f}-S_{21}^{h} \\
& =\left(\Gamma_{1}+\Gamma_{2}\right) e^{-\mathrm{j} \beta L}+\left(\Gamma_{1} \Gamma_{2}\right) e^{-\mathrm{j} \beta L} .
\end{aligned}
$$

Since we are dealing with soft faults, $\left|\Gamma_{i}\right| \ll 1, i=1,2$, so that $\Gamma_{1} \Gamma_{2} \ll \Gamma_{1}+\Gamma_{2}$, allows in turn to simplify the $S_{21}$ expression to

$$
S_{21} \approx\left(\Gamma_{1}+\Gamma_{2}\right) e^{-\mathrm{j} \beta L}=S_{21}^{1}+S_{21}^{2} .
$$

where $S_{21}^{i}$ is the scattering transmission coefficient of the single-fault NUT with only the $i$ th fault appearing, as in Figs. $5(\mathrm{~b}-\mathrm{c})$. The level of accuracy in neglecting the product $\Gamma_{1} \Gamma_{2}$ can be better grasped by taking the special case $\Gamma_{1}=\Gamma_{2}=\Gamma$, yielding $\left|\Gamma_{1} \Gamma_{2}\right| /\left|\Gamma_{1}+\Gamma_{2}\right|=|\Gamma| / 2$. Typical values of $|\Gamma|$ shown in sec. $\mathrm{V}$ are smaller than 0.01 , implying a residual error of less than $1 \%$.

The same result can be found for the remaining terms in the baselined scattering matrix, i.e.,

$$
\boldsymbol{S}_{G} \approx \sum_{i=1}^{N_{f}} \boldsymbol{S}_{i}
$$

where $N_{f}$ is the number of faults in the NUT. This result is in general valid for any layout, as long as the faults can be regarded as soft, a property equivalent to the first Born approximation widely used in wave scattering [19].

The superposition (5) can be exploited in order to estimate the scattering matrix of the $i$ th fault from the measured $\boldsymbol{S}_{G}$ matrix. To this end, let us recall that the SDORT does provide a very good estimate of the position of the faults. In particular, the position of strongest focusing typically coincides with the most severe fault. This observation can serve as a guess of the position of one of the faults. It is therefore possible to insert a dummy fault into the healthy-NUT model, previously used for baselining: the nature of this dummy fault is not important, as discussed later on. The scattering matrix of this modified NUT will be referred to as $S_{d}^{i}$, for the $i$ th fault. This would be the response of the NUT if only one fault was present. The idea is now to remove the contribution of $\boldsymbol{S}_{d}^{i}$ from $\boldsymbol{S}_{G}$, in order to be left with a scattering matrix containing only the remaining $N_{f}-1$ faults.

To this end, we can exploit a fundamental property of SDORT: in case of a single-fault, the scattering matrix has only one eigenvalue. Since each $\boldsymbol{S}_{i}$ matrix is symmetric, it can be expressed exactly as [20].

$$
\boldsymbol{S}_{i}=\nu_{i} \boldsymbol{w}_{i} \boldsymbol{w}_{i}^{\mathrm{T}}
$$

where $\nu_{i}$ is the only non-zero eigenvalue of $\boldsymbol{S}_{i}$ and $\boldsymbol{w}_{i}$ the associated eigenvector, with $T$ is the transpose operator. As shown in [21], the eigenvectors only depend on the position of the fault rather than its intensity, which is coded by $\nu_{i}$. Therefore, assuming the guessed position of the fault is correct, the scattering matrix of the dummy-fault NUT is

$$
\boldsymbol{S}_{d}^{i}=\mu_{i} \boldsymbol{w}_{i} \boldsymbol{w}_{i}^{\mathrm{T}}
$$

i.e., correct up to a scalar factor dependent on the unknown fault reflectivity, implying that $\boldsymbol{S}_{i}=\alpha_{i} \boldsymbol{S}_{d}^{i}$. Hence, from (5),

$$
\boldsymbol{S}_{G}=\sum_{i=1}^{N_{f}} \alpha_{i} \boldsymbol{S}_{d}^{i}=\sum_{i=1}^{N_{f}} \alpha_{i} \mu_{i} \boldsymbol{w}_{i} \boldsymbol{w}_{i}^{\mathrm{T}}
$$

The missing coefficients $\alpha_{i}$ can be estimated by noticing that

$$
\boldsymbol{w}_{i}^{\mathrm{T}} \boldsymbol{S}_{G} \boldsymbol{w}_{i}=\alpha_{i} \mu_{i}\left(\boldsymbol{w}_{i}^{\mathrm{T}} \boldsymbol{w}_{i}\right)^{2}+\sum_{k \neq i} \alpha_{k} \mu_{k}\left(\boldsymbol{w}_{k}^{\mathrm{T}} \boldsymbol{w}_{i}\right)^{2} .
$$

The first term in (9) provides direct access to $\alpha_{i}$. In general, the remainder summation is different from zero when sampled at a given frequency; this is not expected to be an issue, since the scalar products lead to phase-shift angles that change with the frequency. As a result, the time-domain signals used for location involve an averaging effect that is expected to reduce the impact of the remainder, especially for complex networks. 
We will therefore use

$$
\hat{\alpha}_{i}=\frac{\boldsymbol{w}_{i}^{\mathrm{T}} \boldsymbol{S}_{G} \boldsymbol{w}_{i}}{\mu_{i}\left(\boldsymbol{w}_{i}^{\mathrm{T}} \boldsymbol{w}_{i}\right)^{2}}=\alpha_{i}+\sum_{k \neq i} \alpha_{k} \frac{\mu_{i}\left(\boldsymbol{w}_{k}{ }^{\mathrm{T}} \boldsymbol{w}_{i}\right)^{2}}{\mu_{k}\left(\boldsymbol{w}_{k}^{\mathrm{T}} \boldsymbol{w}_{k}\right)^{2}}
$$

as an estimate of $\alpha_{i}$. Notice that (10) is based on the measured baselined scattering matrix of the multiple fault NUT, and the eigenexpansions of the dummy single-fault NUTs.

Finally, (10) yields an estimate of the single-fault scattering matrices, as

$$
\hat{\boldsymbol{S}}_{i}=\hat{\alpha}_{i} \boldsymbol{S}_{i}^{d} .
$$

Access to the individual $\boldsymbol{S}_{i}$ not only allows energy focusing on individual faults, but also estimating the fault severity, as will discussed in sec. IV-C.

\section{B. The EDORT algorithm}

The previous discussion and results can be translated into an algorithm that iteratively extracts the individual scattering matrices of single faults of an NUT. The steps required are summarized in what follows:

1) measure the global scattering matrix $S_{G}^{1}$ of the (eventually) multiple faulty NUT.

2) apply the SDORT method on $S_{G}^{i}$, in order to estimate the position of the strongest soft fault

3) include a dummy soft fault on the guessed position and compute its scattering matrix $\boldsymbol{S}_{d}^{i}$

4) compute the only significant eigenvalue of $\boldsymbol{S}_{d}^{i}$ and its eigenvector

5) compute $\hat{\alpha}_{i}$ and $\hat{S}_{i}$, according to (10) and (11)

6) remove the $i$ th fault contribution by computing $\boldsymbol{S}_{G}^{i+1}=$ $\boldsymbol{S}_{G}^{i}-\hat{\alpha}_{i} \boldsymbol{S}_{d}^{i}$

7) increase $i$ and repeat from step 2) until the update $\boldsymbol{S}_{G}^{i}$ has no significant eigenvalue.

The stop criterion in this algorithm requires to set a threshold for negligible eigenvalues, a choice that really depends on the configuration, especially the noise level, numerical accuracy, etc. To better visualize this point, Fig. 6 shows the dominant eigenvalue of each EDORT iteration applied to the single branch NUT of Fig. 1(b). In fact, two significant eigenvalues corresponding to the first and second iteration can be recognized, whose eigenvector components located the first and second soft fault present in the NUT. On the other hand, the third iteration leads to a negligible eigenvalue compared to the first two thus indicating the absence of any new considerable discontinuity in the structure. For instance, in the case of a purely numerical computation, one could also consider the machine epsilon. Besides from a more practical point of view, as the estimated severity gets very small, the user could stop the procedure as it is no longer worth continuing, since the fault would pose no threat to the proper functioning of the NUT.

\section{Estimating the fault severity}

Having provided an approximate procedure for extracting the single-fault matrices $\boldsymbol{S}_{i}$, the severity of each fault can be assessed without ambiguity, as these matrices have rank equal to one. Since the first Born approximation underpins soft-fault

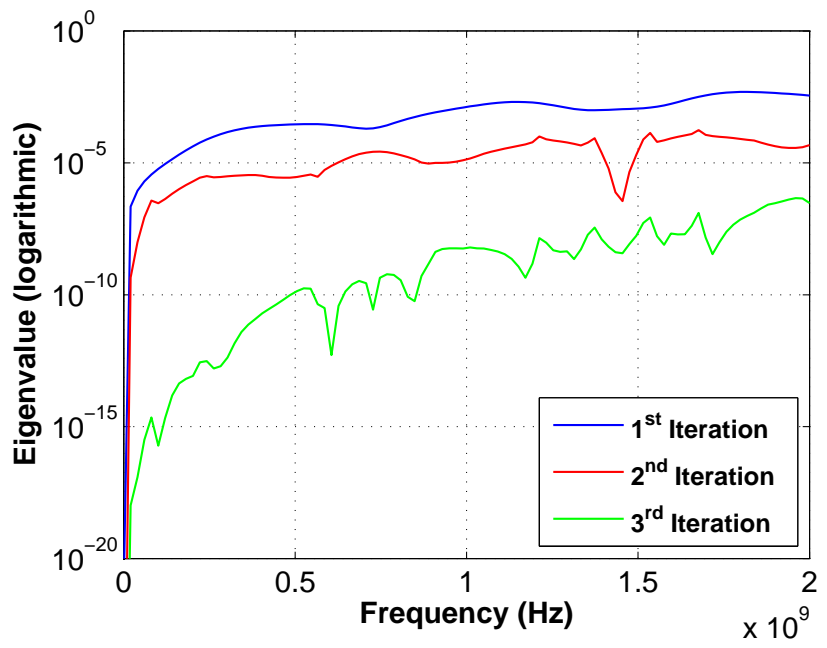

Fig. 6: The most significant eigenvalue for the TRO, calculated for the single junction NUT of Fig. 1(b) after applying the EDORT steps for the fist, second and third iterations.

related scattering matrices, by defining $\tau_{k}$ the transmission function between the fault position and the $k$ th port

$$
S_{k j}=\Gamma_{i} \tau_{k} \tau_{j},
$$

where $\Gamma_{i}$ is the reflection coefficient of the $i$ th fault. Noting as $\left\|\boldsymbol{S}_{i}\right\|_{F}$ the Frobenius norm of $\boldsymbol{S}_{i}$, (12) yields

$$
\left|\Gamma_{i}\right|=\left\|\boldsymbol{S}_{i}\right\|_{F}\left(\sum_{k=1}^{N}\left|\tau_{k}\right|^{2}\right)^{-1} .
$$

This last result requires a priori knowledge of the $\tau_{i}$ functions. In fact, these are known through the healthy NUT model used for baselining. The fact that only the moduli of the $\tau_{i}$ are required in (13) implies that errors in the positioning of the dummy fault have a minor impact on the accuracy of the estimated $\left|\Gamma_{i}\right|$, as long as the NUT is tested at frequencies where propagation can be regarded as subject to weak dissipation.

Departure from Born first approximation are shown in sec. V-B to lead to local errors that have in fact negligible impact on the overall performance of the procedure, as these errors have zero average (i.e., turning phase-shift angles).

\section{VALIDATION}

The EDORT method is tested against experimental data collected according to the setup described in sec. V-A. After showing that the ability of testing each fault separately is reinstated, the accuracy of the estimation of the fault severity is tested in sec. V-B.

\section{A. Experimental results}

Experimental tests were conducted on the NUT configurations in Figs. 1(a)-(b), implemented using standard $50 \Omega$ coaxial cables as transmission lines. Although coaxial cables are not routinely found in practical NUTs, they were chosen 
because of their excellent stability, in order to ensure reproducible results. The conclusions drawn in this section are not related to the type of lines used, since the proposed method is completely general.

Coaxial cables also offer another sizeable advantage, since soft faults can be introduced (and removed) by using a set of $30-\mathrm{cm}$ long samples made of semi-rigid coaxial lines, as those shown in Fig. 7. Each sample was crushed by a different force over a different portion of length, thus producing a varying severity in the faults: the strongest soft fault is designated by a crushed length of $2 \mathrm{~cm}$ while the weaker one by $1 \mathrm{~cm}$. This same approach was used in the experimental validation of the standard DORT done in [15], where it allowed a practical and reproducible framework. In fact, the direct generation of the faults in a transmission line is hardly reproducible, not allowing a double check of results due to the fact that it is an irreversible process.

The ends of the cables were used as testing ports, by connecting them to a Rohde \& Schwarz ZVB8 VNA, capable of covering a frequency range going from $300 \mathrm{kHz}$ to $8 \mathrm{GHz}$ with four testing ports. The measurement of the scattering matrices was done over a total bandwidth of $2 \mathrm{GHz}$ and a frequency step of $20 \mathrm{MHz}$. After calibrating the VNA using the calibration kit provided by the manufacturer, studying the network consisted of two steps:

1) measurement of $\boldsymbol{S}_{G h}$ of the reference healthy system (without faults), where we considered the network with unaltered $30-\mathrm{cm}$ semi-rigid sections.

2) measurement of $\boldsymbol{S}_{G f}$ of faulty systems considering the introduction of the two soft faults on their positions as presented in the configurations of Fig. 1(a)\&(b), where the unaltered cable samples were replaced by the faulty ones.

Having measured the two scattering matrices for each NUT, the algorithm detailed in sec. IV-B was applied. Signals propagating at each step throughout the NUT were computed by means of an in-house transmission-line solver, implemented under Matlab.

The proposed procedure was first applied to the singlebranch NUT in Fig. 1(a) with two soft faults on $1.15 \mathrm{~m}$ and 2 $\mathrm{m}$ from the reference port, and implemented as shown in Fig. 8. The energy diagrams associated to the estimated singlefault scattering matrices in Fig. 9 are now characterized by a single dominant peak each, corresponding to the position of the two faults. These results show the result of selective focusing compared to the that obtained by the SDORT on the same NUT, featuring two peaks simultaneously on the faults' positions as presented also in Fig. 8.

It can be noticed that some artifacts are still present, due to the periodicity of wave propagation that is proper of singlebranch structures. Indeed, this problem disappears as soon as more complex structures are considered, where the spatial periodicity is broken. The flat background observed in the two energy distributions can be understood by recalling that, in the case of single faults, excitation signals with equivalent intensity cross the NUT and interfere constructively at the fault position [15].

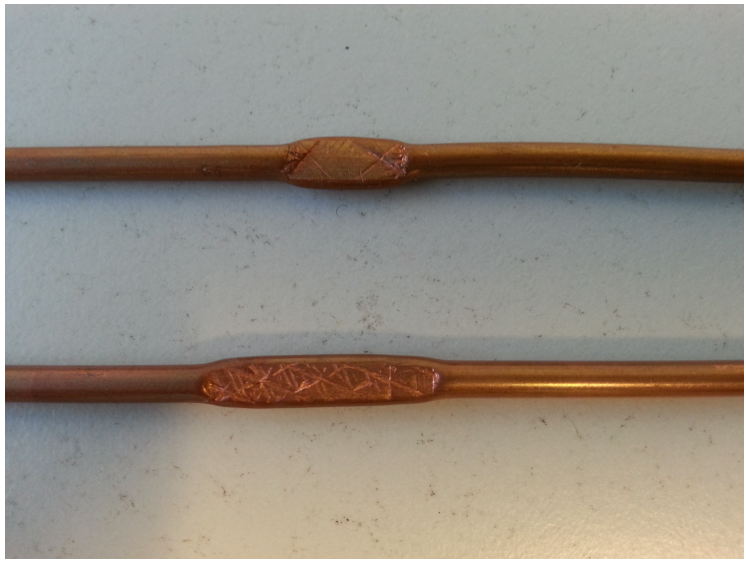

Fig. 7: The two faulty samples used for introducing soft faults in experiments.

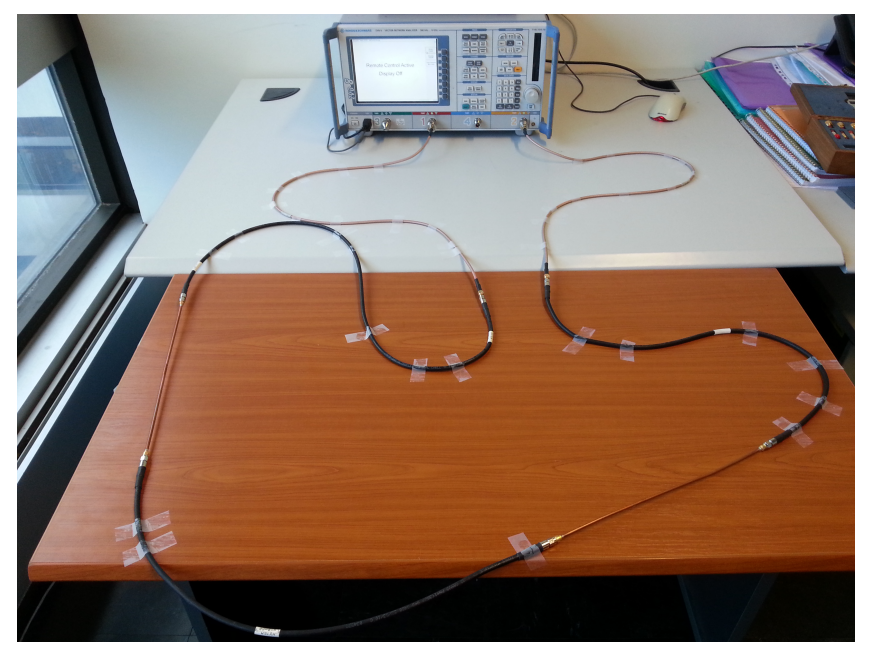

Fig. 8: Experimental setup for the cable NUT of Fig. 1(a), containing two faulty sections $(1.15 \mathrm{~m}$ and $2 \mathrm{~m}$ from the reference port), connected to the VNA for the experimental tests.

In order to check the efficiency of the proposed method with more complex NUTs, the single-junction structure in Fig. 1(b) was considered and implemented as presented in Fig. 10 considering the first soft fault at $0.8 \mathrm{~m}$ from the reference port and the weaker at $2.4 \mathrm{~m}$ on the second path. Indeed, in this case artifacts are negligible with respect to the focusing peaks. The interest of applying the proposed procedure becomes clear when comparing the EDORT results in Fig. 11 with those from SDORT, shown in the same figure, where artifacts would have led to a wrong conclusion about the faults position. The separation of the fault responses yields similar features for the two faults, independently of their relative severity.

\section{B. Retrieving fault reflection coefficients}

In order to assess the accuracy of (13) in retrieving reflection coefficients of the faults, their values were measured by directly connecting the faulty samples to one port of the VNA, while terminating the remaining port of the samples with a 


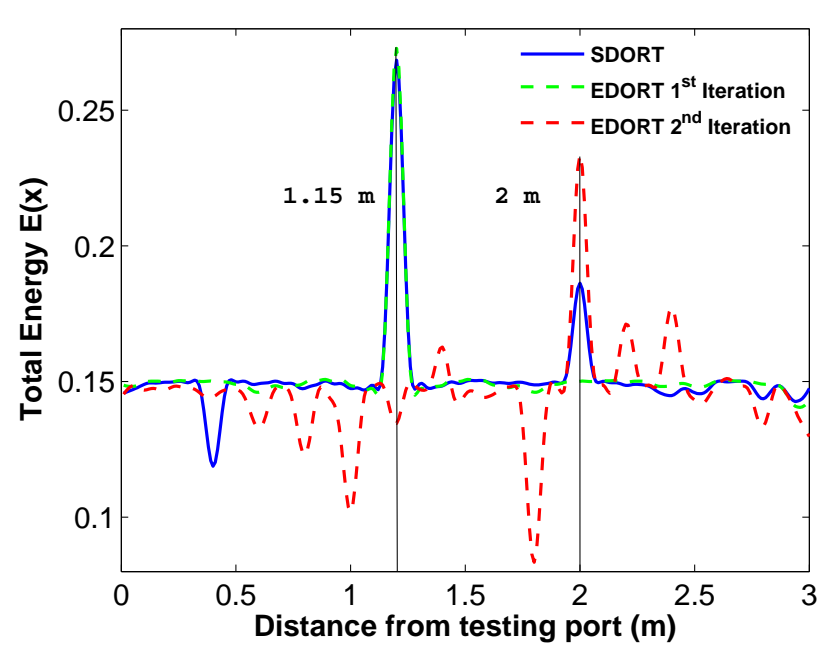

Fig. 9: The total energy along the NUT in Fig. 1(a), computed after applying the EDORT separation procedure. At each iteration the dominant energy peak corresponds to a fault not identified by the previous iterations. The energy distribution obtained when using the SDORT is also shown for reference, and contains two energy peaks at the position of the two faults.

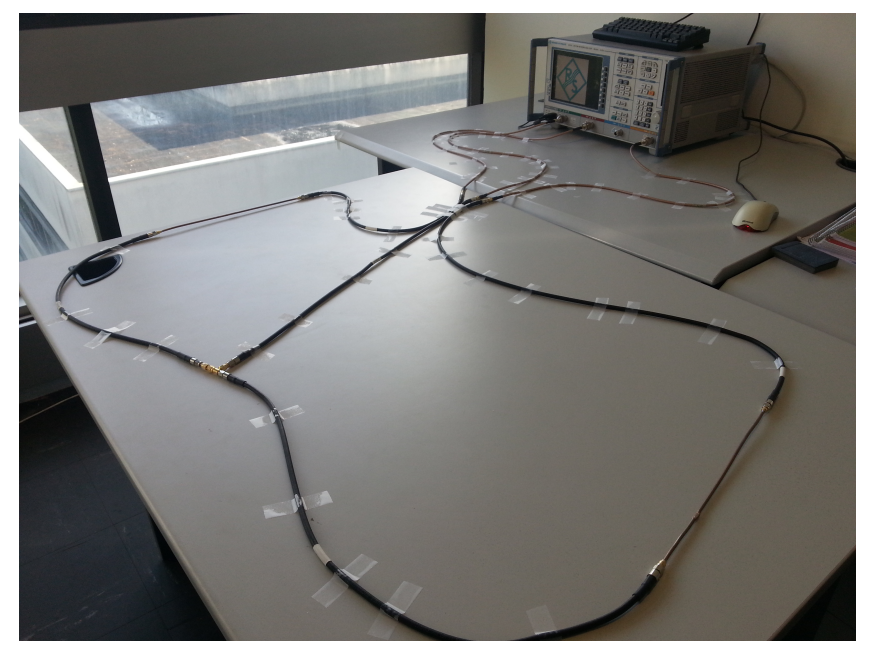

Fig. 10: Experimental setup for the single-junction NUT in Fig. 1(b) presenting two faulty sections $(0.8 \mathrm{~m}$ and $2.4 \mathrm{~m}$ from the reference port), connected to the VNA for the experimental tests.

matched load. The resulting reflection coefficients are shown in Fig. 12. Eq. (13) was subsequently applied to the previously estimated single-fault scattering matrices. Results are shown in Fig.12, for the single-branch NUT of Fig. 8. The two sets of data closely agree.

The same operations were then carried out for the singlejunction NUT in Fig. 10. In this case, we can expect interactions between the faults and the discontinuity introduced by the junction. As a result, the simple model proposed in (12) is expected to hold only on average. Fig. 13 shows the comparison between the measured and (13)-based estimates of

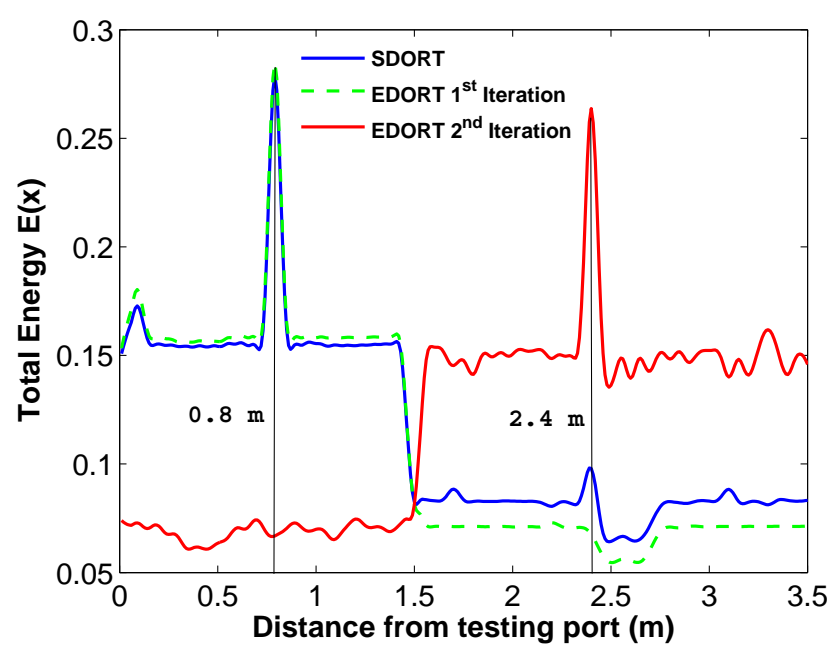

Fig. 11: Same kind of results shown in Fig. 9, but now dealing with the single-junction NUT in Fig. 1(b).

the reflection coefficients. It appears that the estimates oscillate around the true values that should have been retrieved.

Since this kind of behaviour can be expected to have zeroaverage, because of phase rotation associated to frequency variations introduced by propagation delay, a simple linear regression through the estimate from (13) can be applied in order to reduce the impact of these oscillations. The results in Fig. 13 confirm the effectiveness this procedure. Both reflection coefficients agree with the direct measurements.

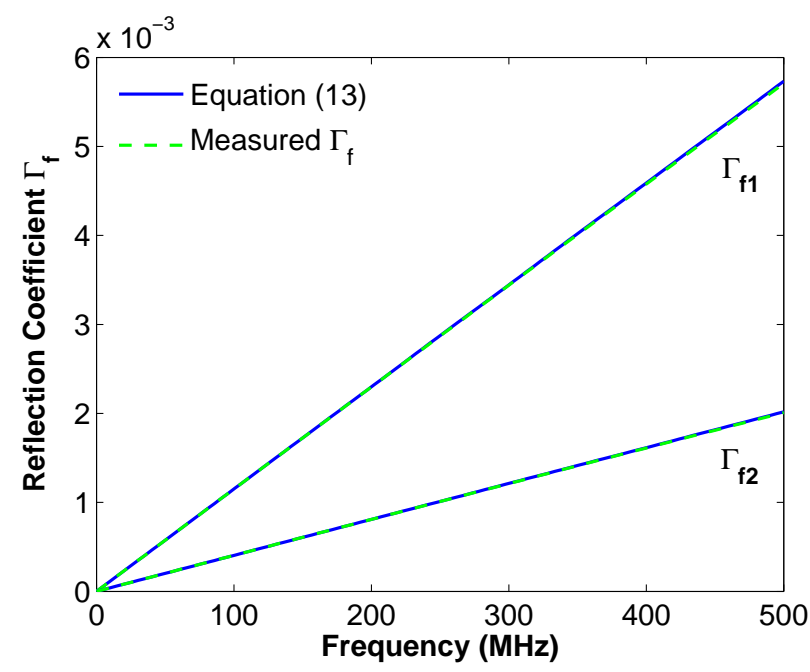

Fig. 12: Amplitude of the measured reflection coefficients of the two faulty samples in Fig. 7 versus frequency. Estimates of these reflection coefficients obtained by means of the EDORT and (13) applied to experimental data from VNA measurements are also shown, corresponding to the setup in Fig. 8. 


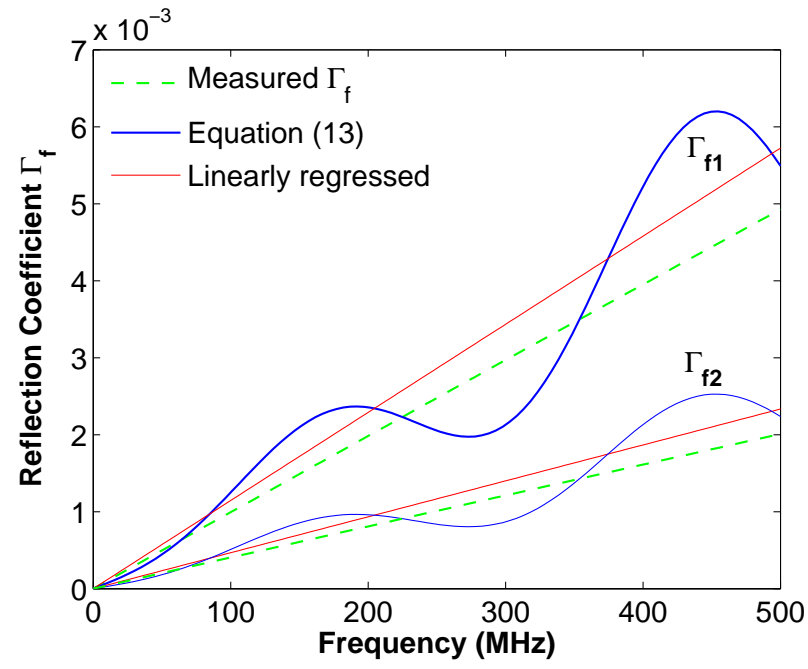

Fig. 13: Same kind of results as in Fig. 12, but now dealing with the experimental setup in Fig.10. The results estimated by means of (13) are here also used as the basis for a linear regression, as explained in the body of the text.

\section{Conclusions}

Having pointed out the shortcomings of SDORT in the case of multiple soft faults, an alternative formulation based on an updating scheme was presented. The input signals used for imaging the faults were shown to focus selectively on single faults, allowing a clear identification. No spurious focusing was observed, thus reducing the probability of false alarms. Experimental validations also confirmed these conclusions, when considering both very weak faults and non-trivial NUT layouts. These results give credibility to the practical usefulness of the proposed method in critical real-life configurations that are hardly addressed in current literature. In addition to that, the proposed procedure allows an accurate estimate of the severity of each fault, a feature of practical interest when monitoring the state of critical cable networks.

Future work will need to deal with the robustness of EDORT with respect to errors in the baselining procedure, as due, e.g., to tolerances in the geometrical description of the reference healthy network.

\section{REFERENCES}

[1] C. Furse and R. Haupt, "Down to the wire [aircraft wiring]," Spectrum, IEEE, vol. 38, no. 2, pp. 34-39, 2001.

[2] F. Auzanneau, "Wire troubleshooting and diagnosis: Review and perspectives," Progress In Electromagnetics Research B, vol. 49, pp. $253-$ 279, 2013.

[3] L. A. Griffiths, R. Parakh, C. Furse, and B. Baker, "The invisible fray: A critical analysis of the use of reflectometry for fray location," Sensors Journal, IEEE, vol. 6, no. 3, pp. 697-706, 2006.

[4] Y.-J. Shin, E. J. Powers, T.-S. Choe, C.-Y. Hong, E.-S. Song, J.-G. Yook, and J. B. Park, "Application of time-frequency domain reflectometry for detection and localization of a fault on a coaxial cable," Instrumentation and Measurement, IEEE Transactions on, vol. 54, no. 6, pp. 2493-2500, 2005.

[5] T.-W. Pan, C.-W. Hsue, and J.-F. Huang, "Time-domain reflectometry using arbitrary incident waveforms," Microwave Theory and Techniques, IEEE Transactions on, vol. 50, no. 11, pp. 2558-2563, 2002.

[6] S. J. P., Time-domain Reflectometry for Monitoring Cable Changes: Feasibility Study. Electric Power Research Institute, 1990.
[7] C. P. Nemarich, "Time domain reflectometry liquid level sensors," Instrumentation \& Measurement Magazine, IEEE, vol. 4, no. 4, pp. 4044, 2001.

[8] N. Paulter, "An assessment on the accuracy of time-domain reflectometry for measuring the characteristic impedance of transmission lines," Instrumentation and Measurement, IEEE Transactions on, vol. 50, no. 5, pp. 1381-1388, 2001

[9] C. Furse, Y. C. Chung, R. Dangol, M. Nielsen, G. Mabey, and R. Woodward, "Frequency-domain reflectometry for on-board testing of aging aircraft wiring," Electromagnetic Compatibility, IEEE Transactions on, vol. 45, no. 2, pp. 306-315, 2003.

[10] Y. C. Chung, C. Furse, and J. Pruitt, "Application of phase detection frequency domain reflectometry for locating faults in an f-18 flight control harness," Electromagnetic Compatibility, IEEE Transactions on, vol. 47, no. 2, pp. 327-334, 2005.

[11] C. Furse, Y. C. Chung, C. Lo, and P. Pendayala, "A critical comparison of reflectometry methods for location of wiring faults," Smart Structures and Systems, vol. 2, no. 1, pp. 25-46, 2006.

[12] Q. Zhang, M. Sorine, and M. Admane, "Inverse scattering for soft fault diagnosis in electric transmission lines," Antennas and Propagation, IEEE Transactions on, vol. 59, no. 1, pp. 141-148, 2011.

[13] H. Boudjefdjouf, R. Mehasni, A. Orlandi, H. Bouchekara, F. De Paulis, and M. Smail, "Diagnosis of multiple wiring faults using time-domain reflectometry and teaching-learning-based optimization," Electromagnetics, vol. 35, no. 1, pp. 10-24, 2015.

[14] C. Prada and M. Fink, "Eigenmodes of the time reversal operator: A solution to selective focusing in multiple-target media," Wave motion, vol. 20, no. 2, pp. 151-163, 1994.

[15] L. Abboud, A. Cozza, and L. Pichon, "A noniterative method for locating soft faults in complex wire networks," Vehicular Technology, IEEE Transactions on, vol. 62, no. 3, pp. 1010-1019, 2013.

[16] A. Lelong, L. Sommervogel, N. Ravot, and M. O. Carrion, "Distributed reflectometry method for wire fault location using selective average," Sensors Journal, IEEE, vol. 10, no. 2, pp. 300-310, 2010.

[17] G. Cerri, R. De Leo, L. Della Nebbia, S. Pennesi, V. M. Primiani, and P. Russo, "Fault location on shielded cables: Electromagnetic modelling and improved measurement data processing," IEE Proceedings-Science, Measurement and Technology, vol. 152, no. 5, pp. 217-226, 2005.

[18] C. R. Paul, Analysis of multiconductor transmission lines. John Wiley \& Sons, 2008

[19] M. Born and E. Wolf, Principles of optics: electromagnetic theory of propagation, interference and diffraction of light. Cambridge university press, 1999.

[20] R. A. Horn and C. R. Johnson, Matrix analysis. Cambridge university press, 2012.

[21] C. Prada, S. Manneville, D. Spoliansky, and M. Fink, "Decomposition of the time reversal operator: Detection and selective focusing on two scatterers," The Journal of the Acoustical Society of America, vol. 99, no. 4, pp. 2067-2076, 1996. 